\title{
The Use of Artificial Intelligence Methods in the Evaluation of Medical Data
}

\author{
Gerard Pasternak $^{1 *}$, Katarzyna Pentos ${ }^{2}$, Katarzyna Gul ${ }^{1}$ and Aleksandra Lewandowicz-Uszynska ${ }^{1}$ \\ ${ }^{1}$ Department of Immunology and Pediatrics, Poland \\ ${ }^{2}$ Institute of Agricultural Engineering, Poland
}

*Corresponding author: Gerard Pasternak, Department and Clinic of Paediatrics, Immunology and Rheumatology of Developmental Age, Poland

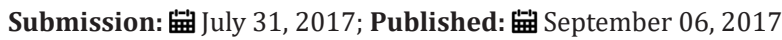

\section{Introduction}

The $20^{\text {th }}$ century brought a very dynamic development of medicine as well as other fields of science. Leonard Hay flick writes towards the end of the $20^{\text {th }}$ century in his book "How and Why We Age In 1900, as many as 75 percent of US citizens did not survive sixty-five years. Today, these statistics have almost reversed: about 70 percent live more than sixty-five years. What caused such a significant increase in the average life expectancy? Hay flick explains that this should be attributed "primarily to the decline in infant mortality"[1]. Obviously this is a very important factor in raising the population's life expectancy. Furthermore, it must be born in mind that both, life expectancy and quality of life are influenced by a whole array of environmental factors. Therefore, it is extremely important to be influenced by the most recent achievements in broadly understood medicine. First of all, patient care based on evidence-based medicine [2-5].

However, scholar's ambitions go far beyond providing basic health care [6]. Today's technology is revolutionizing medicine. It is believed that scientists double the medical knowledge every eight years. We get acquainted with some of the recent technological developments as well as actions with a view to helping to eradicate the disease [7]. Is science able to provide people with satisfactory health condition? Is it able to achieve such an objective at the beginning of the $21^{\text {st }}$ century?

Technological progress makes it possible to perform even complex calculations in significantly less time. With the discovery of effective medicines, a list of diseases initially seemed incurable has been narrowed with the direct effect on the increasing number of people in the world as well as the improvement of the state of their health. There were a lot of discoveries due to which new cancer therapies were possible, what leads us to hope that this particular disease will no longer gather its fatal harvest [8].

The most successful achievements of the $21^{\text {st }}$ century medicine should include new medicines to fight AIDS. Researchers are also getting closer to discovering an effective HIV vaccine. Also, subsequent discoveries in genetics raise our hopes of possible effective treatments for initially incurable diseases creating [9].

In the $21^{\text {st }}$ century we derive from the knowledge gained in the last century, aiming to obtain increasingly better diagnostic and therapeutic methods [10]. Year by year the number of lab tests is increasing. The results of these studies can be considered as scientific evidence if only we could determine and measure the effectiveness and costs of individual medical procedures. Every patient admitted to hospital diagnostics has a routinely performed panel of tests consisting of an average of 25-35 different laboratory tests. For example, complete blood count and white cell differential includes 18 parameters. Assessing the number of results from one patient and multiplying it by a group of 100 , for example, gives a great database, interpretation of which would be very fiddle. Clinical analysis should include first-class scientific evidence and the reliability of its results depends on the proper acquisition and methodology for the development of the final results. Undoubtedly, the share of artificial intelligence in the process of analyzing large amounts of data remains significant.

Among many soft computing techniques used for medical data analysis, artificial neural networks (ANNs) play very important role. ANNs are inspired by human nervous system. The main element of ANNs is artificial neuron, fundamental information-processing unit, which is very simple equivalent of natural neuron. ANNs consist of many artificial neurons composed in layers. Neurons in one layer are connected with those in the adjacent layers and these connections are described by "weights". The ANNs employment doesn't require the knowledge about relationship under study. ANN is trained with the use of experimental data set and during training process, "weights" are adjusted in order to decrease the difference between output signal calculated by ANN and expected value of this signal known from training data set. ANNs are considered as an effective substitute for the classical empirical and statistical process modeling techniques and are successfully used as models of complex and nonlinear relationships. 
Rigorous literature survey, indicates that ANNs are widely used in many fields of medicine. ANN-based model was proposed for the diagnosis of obstructive sleep apnea(OSA), based on easily acquired patient demographic data including sex, age, body mass index, and snoring status. With the model, the detection of OSA was possible within few seconds with the accuracy of $86.6 \%$. Developed decision support system for the prediction of the case of peripheral nerve disorder. Their work contained also a classifier for distinguishing between carpal tunnel syndrome, neuropathy and normal peripheral nerve conduction. To this aim, two types of ANN were employed: recurrent neural network and a two-layer feed forward network. Moreover, the system was equipped with graphical user interface. Recurrent neural network performed better as classification tool, with the accuracy of $98.6 \%$ and the feed forward ANN was able to achieve an accuracy of $97.4 \%$. The Multilayer Feed forward Neural Network was chosen by as machine-learning technique for developing the diagnosis of lung cancer support system. The lung cancer identification was based on sympathetic skin response measurements with 100\% accuracy. Lung cancer diagnosis and patients treatment supported by ANNs was the subject of more papers published recently. The feed forward ANN with architecture 25-4-1 was used by for prediction of survival time in advanced lung cancer as affected by the genetic polymorphism of the $\mathrm{p} 21$ and $\mathrm{p} 53$ genes in conjunction with patients general data. The prediction accuracy of the model was $86.2 \%$. Additionally, authors assessed the importance of explanatory variables in the model. They concluded that the most important input variable was the clinical staging of lung cancer patients, and the next most important variables were pathological type and responsiveness to treatment. The model for prediction of the risk of lung cancer based on BP Neural Networks proposed by [10] provided satisfactory accuracy under low dimensions of risk factors. The ANN model with the six serum tumor markers and 19 other parameters was described by [5]. The aim of ANN employment was to distinguish lung cancer from benign lung disease and healthy people. Authors claimed the follows parameters of the model: sensitivity $98.3 \%$, the specificity $99.5 \%$ and the accuracy $96.9 \%$. However, as was underlined by [3] the use of ANN presented in the medical literature is not always performed in an accurate manner. In the effect, reliability of the results presented raises doubts.
It can be stated that modern soft computing techniques can be widely used in the medical, health, and pharmacy fields, where the analysis of large, complex data is required. In particular, when taking into account precision medicine, which relies on an increasing amount of heterogeneous data. Clinicians need to use many diagnostic tools including medical imaging, blood tests, and genomics in order to decide the appropriate combination of treatments such as radiotherapy, chemotherapy, targeted therapy and immunotherapy [4] For that reason, more and more data need to be analyzed and therefore sophisticated artificial intelligent methods are developed and employed by researchers. This trend is obvious when the aim is better healthcare.

\section{References}

1. Hay flick L (1998) How and why we age. Exp Gerontol 33(7-8): 639-653.

2. Masic I, Miokovic M, Muhamedagic B (2008) Evidence based medicinenew approaches and challenges. Acta Inform Med 16(4): 219-25.

3. Bertolaccini L, Solli P, Pardolesi A, Pasini A (2017) An overview of the use of artificial neural networks in lung cancer research. Journal of Thoracic Disease 9(4): 924-931.

4. Bibault JE, Giraud P, Burgun A (2016) Big data and machine learning in radiation oncology: State of the art and future prospects. Cancer Letters 382(1): 110-117.

5. Feng FF, Wu YM, Wu YJ, Nie GJ, Ni R, et al. (2012) The effect of artificial neural network model combined with six tumor markers in auxiliary diagnosis of lung cancer. Journal of Medical Systems 36(5): 2973-2980.

6. Hsia TC, Chiang HC, Chiang D, Hang LW, Tsai FJ, et al. (2003) Prediction of survival in surgical un resectable lung cancer by artificial neural networks including genetic polymorphisms and clinical parameters. Journal of Clinical Laboratory Analysis 17(6): 229-234.

7. Karamanli H, Yalcinoz T, Yalcinoz MA (2016) A prediction model based on artificial neural networks for the diagnosis of obstructive sleep apnea. Sleep and Breathing 20(2): 509-514.

8. Kunhimangalam R, Ovallath S, Joseph PK (2013) Computer aided diagnostic problem solving: Identification of peripheral nerve disorders. Irbm 34(3): 244-251.

9. Oezkan O, Yildiz M, Bilgin S, Koklukaya E (2010) Measuring the sympathetic skin response on body and using as diagnosis-purposed for lung cancer patients by artificial neural networks. Journal of Medical Systems 34(3): 407-412.

10. Xie NN, Hu L, Li TH (2014) Lung cancer risk prediction method based on feature selection and artificial neural network. Asian Pacific Journal of Cancer Prevention 15(23): 10539-10542. 